\title{
Preparation and Properties of Thermotropic Mesogenic Polyesters
}

\author{
Leszek MAKARUK, Hanna POLAṄSKA, Elżbieta KSIĘŻAKOWSKA, \\ and Barbara WAŻYṄSKA* \\ Department of Chemistry, Technical University (Politechnika), \\ Noakowskiego 3, 00-662 Warsaw, Poland \\ *Institute of Materials Science and Engineering, \\ Technical University (Politechnika), \\ Narbutta 85, 02-524 Warsaw, Poland
}

(Received December 26, 1984)

\begin{abstract}
The effect of the chemical structure and stereogeometry of mesogen on the liquid crystalline properties of thermotropic main-chain polyesters was studied by means of DSC and polarized microscopy for three series of polymers. The polyesters obtained from modified $4,4^{\prime}-$ dihydroxybenzophenone (DHBP) and sebacoyl (SEB) or suberoyl (SUB) dichlorides did not exhibit the mesogenic properties. Modification of DHBP in this case consisted in replacement of ketone linkage by $-\mathrm{C}_{\mathrm{CC}}^{-}$between two aromatic rings. The same bisphenol, however, when mixed with another (showing mesogenic behaviour) enhanced the liquid crystalline properties of the copolyesters, namely increasing the temperature range of liquid crystalline phase. The condensation of SEB or SUB with DHBP modified by lateral substitution (at various positions) of one aromatic ring with $\mathrm{CH}_{3}$ group lead to polyesters of lower temperatures of the transition from crystal to nematic phase and mesophase to isotropic liquid, as compared to parent polymers (i.e., polyesters of DHBP $+\mathrm{SEB}$ or SUB). Different shifts of those temperatures with the position of substitution have been observed and it was found less pronounced for 3-substituted than 2-substituted bisphenols. Temperature ranges of mesophases also depended on the structure of bisphenols and were broader for 3substituted DHBP. Therefore, the stereogeometry of the polymer molecules and the bulkiness of their substituents are considered the most important parameters in controlling the mesogenic properties of thermotropic, rigid-flexible polyesters with mesogens incorporated in the main chain.

KEY WORDS Thermotropic Liquid Crystalline Polyesters and Copolyesters / Main-Chain Mesogenic Polyesters / Rigid Flexible Polyesters / Laterally Modified Bisphenol Mesogens / DSC Studies / Melting and Clearing Temperatures /
\end{abstract}

In recent years the liquid crystalline polymers have drawn much attention mainly due to their excellent mechanical properties. There are, however, still lot of questions which are to be answered. Principally, they all concerned relationship between the structure and mesogenic properties of polymeric materials, the characteristics which are already fairly well known for low molecular weight liquid crystals.

From the recently published very systematic reports by Blumstein ${ }^{1}$ and Lenz, ${ }^{2}$ the following factors affecting the type and stability of mesophase of thermotropic, rigid-flexible, main-chain polymers can be drawn: 1 . the chemical structure and stereogeometry of the macromolecular chain, 2. the lengths of the rigid (mesogenic) and flexible segments as well as the type of the group linking those units together, 3. the molecular weight and the molecular weight distribution of the polymer. 
In our earlier papers ${ }^{3,4}$ we have presented results concerning the effect of the lengths of rigid and flexible segments on the transition temperatures and mesophasic range of copolyesters and copolyamidoesters obtained from dicarboxylic acid dichlorides and derivatives of 4,4'-dihydroxybenzophenone (DHBP).

In the present paper we further discuss those effects but we will mainly concentrate on the influence of the structure and stereogeometry of the mesogenic unit on the liquid crystalline properties of the resulting polymer.

The lateral substitution of the aromatic ring of mesogen is expected to broaden the molecule and make the spontaneous packing more difficult due to the steric hindrances or locking of the macromolecular chains. As a result, the decrease of transition temperatures should be encountered.

The above mentioned phenomena have been observed by many authors. ${ }^{5-8}$ Moreover, depending on the position of substitution in aromatic ring, ${ }^{5}$ the type of the substituent (polar or non-polar atoms) ${ }^{6}$ or the fact whether the substitution was symmetric or unsymmetric, ${ }^{7}$ the obtained polymers exhibited quite different characteristics, with the loss of liquid crystallinity at the extreme case. ${ }^{7}$

One should have in mind also, that the broadening of the macromolecules by lateral substitution gives some advantages and sometimes is made intentionally, specifically to lower the melting temperature of potentially mesophasic polymer below the decomposition point. ${ }^{9.10}$ It is therefore, an easy and useful tool in modelling the properties of liquid crystalline polymers.

In our work the modification of polyesters of general formula:

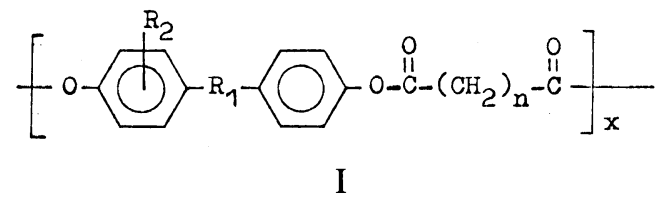

where $n=6$ or 8 was performed.

Studies by means of molecular models showed that the replacement of ketone linkage $\left(\mathrm{R}_{1}\right)^{3,4}$ by $-\underset{\mathrm{C} C \mathrm{Cl}_{2}}{\mathrm{C}}-$ makes the molecule more rigid. Therefore, this should facilitate the regular packing of macromolecules, leading to the increase of the transition temperatures as well as the temperature range of liquid crystalline phase.

On the other hand, the modification of polymer I with methyl substitution of the aromatic ring of the mesogen $\left(\mathrm{R}_{2}=-\mathrm{CH}_{3}\right.$; $\mathrm{R}_{1}=-\underset{\mathrm{O}}{\mathrm{C}-\text { ) }}$ in position 2 or 3 would cause the opposite effect, that is the decrease of transition temperatures and alteration (probably narrowing) of the mesophase range.

In order to confirm our considerations three series of polyesters and copolyesters were prepared:

1. Series $I$, in the reaction of the mixture of DHBP and 2,2'-bis(4-hydroxyphenyl)-1,1dichloroethylene (BDCE, formula II) with se-

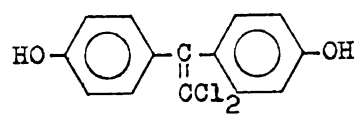

II

bacoyl dichloride (SEB).

2. Series II and III, in the copolycondensation of dichlorides mixture (sebacoyl and suberoyl, SUB) with either 4, 4' - dihydroxy-3methylbenzophenone (DMeBP-3) or $4,4^{\prime}$ dihydroxy-2-methylbenzophenone (DMeBP-2) of the formulae presented below:

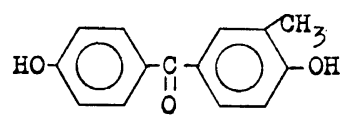

DMeBP-3 (III)

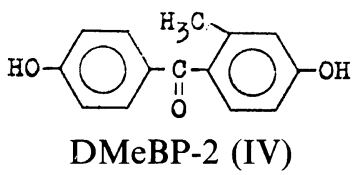




\section{EXPERIMENTAL}

Materials and Monomer Synthesis comonomer in polycondensation reaction was obtained from Merck-Schuchardt and was not further purified. It has the melting point of $214^{c} \mathrm{C}$.

2,2'-Bis(4-hydroxyphenyl)-1,1-dichloroethylene (BDCE) was synthesized from phe-
4,4'-Dihydroxybenzophenone (DHBP), a

nol and chloral hydrate followed by the reaction with aqueous solution of $\mathrm{KOH} .{ }^{11}$ The yield of BDCE was $72 \%$ of the theoretical one and the melting point after the crystallization from $40 \mathrm{vol} \%$ of aqueous solution of ethanol was $215-217^{\circ} \mathrm{C}$ (lit. ${ }^{11} \mathrm{mp}=216-217^{\circ} \mathrm{C}$ ).

4,4'-Dihydroxy-2-methylbenzophenone (DMeBP-2) and 4,4'-dihydroxy-3-methylbenzophenone (DMeBP-3) were obtained following the schemes ${ }^{12,3}$ :

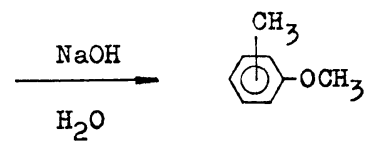

V

VI

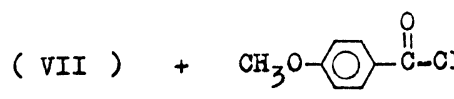

VIII

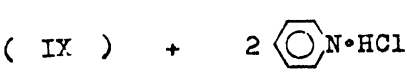

$\mathrm{X}$

To our knowledge those two compounds were so far not described in the literature. In the available references only the preparation of 4,4'-dimethoxy-3-methylbenzophenone (IX), the intermediate product in the synthesis of DMeBP-3, was cited ${ }^{13}$ However, the method of its synthesis was slightly different. The detailed description of DMeBP-2 and DMeBP-3 syntheses will be published elsewhere. $^{14}$

DMeBP-2 in the shape of white needles had a melting point of $86-87^{\circ} \mathrm{C}$. Anal. Found: C, $73.63 \%$; H, 5.64\%; O, 20.73\%. Expected values: C, $73.66 \% ; \mathrm{H}, 5.31 \% ; \mathrm{O}, 21.03 \% .{ }^{1} \mathrm{H}$ NMR: $\delta 9.18(\mathrm{~m}, \mathrm{H},-\mathrm{OH}), 7.26(\mathrm{~m}, 4 \mathrm{H}$, $\left.\mathrm{C}_{6} \mathrm{H}_{4}-\right)$, $6.87\left(\mathrm{~m}, 3 \mathrm{H}, \mathrm{C}_{6} \mathrm{H}_{3}-\right.$ ), and $2.23 \mathrm{ppm}$ (s, $3 \mathrm{H}, \mathrm{CH}_{3}-\mathrm{C}_{6} \mathrm{H}_{3}-$ ). IR: 3500 (s, $\left.-\mathrm{OH}\right), 3300$ (s,
-OH), 1640 (s), 1600 (s), 1580 (s, (s), $1300(\mathrm{~s}), 1240(\mathrm{~s}), 1170(\mathrm{~s}), 1155$ (s), 850 (s), 825 (s), and $740 \mathrm{~cm}^{-1}(\mathrm{w})$.

DMeBP-3 was a pale-purple crystalline precipitate and melted at $227-228^{\circ} \mathrm{C}$. Anal. Found: C, 73.65\%; H, 5.42\%; O, 20.93\%. Expected values: the same as above. ${ }^{1} \mathrm{H}$ NMR: $\delta 9.18(\mathrm{~m}, \mathrm{H},-\mathrm{OH}), 7.51\left(\mathrm{~m}, 3 \mathrm{H}, \mathrm{C}_{6} \mathrm{H}_{3}-\right), 7.26$ $\left(\mathrm{m}, 4 \mathrm{H}, \mathrm{C}_{6} \mathrm{H}_{4}-\right)$, and $2.21 \mathrm{ppm}\left(\mathrm{s}, 3 \mathrm{H}, \mathrm{CH}_{3}-\right.$ $\mathrm{C}_{6} \mathrm{H}_{3}-$ ). IR: 3350 (s, $\left.-\mathrm{OH}\right), 1600$ (s, $\underset{\mathrm{O}}{-\mathrm{C}-}$ ), 1550 (s), 1510 (s), 1315 (s), 1290 (s), 1170 (s), 1150 (s), 1110 (s), 970 (s), 870 (w), 850 (s), $820(\mathrm{w}), 780(\mathrm{~s})$, and $700 \mathrm{~cm}^{-1}$ (s).

Suberoyl and sebacoyl dichlorides were prepared and purified following the procedures 
given in ref 3.

All solvents were purified and stored according to the details described in ref 15 .

\section{Polymer Preparation}

Polyesters were prepared by interfacial polycondensation according to the general procedure given below. In $250 \mathrm{ml}$ reaction flask fitted with double surface reflux condenser, thermometer, dropping funnel and an efficient mechanical stirrer, $0.01 \mathrm{~mol}$ of bisphenol (or mixture of bisphenols) dissolved in $100 \mathrm{ml}$ $1 \mathrm{wt} \%$ aqueous solution of sodium hydroxide and $0.2 \mathrm{~g}$ of benzyltriethylammonium chloride (TEBA) were placed. To a vigorously stirred mixture the proper amount of dicarboxylic acid dichloride (or mixture of dichlorides) in $100 \mathrm{ml}$ of dichloromethane was slowly added (within $30 \mathrm{~min}$ ) from the dropping funnel. After additional two hours of stirring, the organic layer was separated and precipitated while pouring slowly to vigorously stirred $800 \mathrm{ml}$ of methanol. The white or slightly creamy powdered precipitate was filtered, washed with water and methanol and finally dried in vacuum at room temperature.

So-obtained polymers were soluble in dichloromethane, chloroform, less in benzene and its chloro- and nitro-derivatives, and were insoluble in such common solvents as $\mathrm{CCl}_{4}$, acetone, ethyl ether, alcohols (methyl, ethyl).

\section{Methods of Characterization}

Elemental analyses of new monomers and polymers containing chlorine atoms were performed on Perkin-Elmer Model 240 microanalyzer.

Infrared spectra of some polyesters and all monomers in $\mathrm{KBr}$ pellets were obtained with Specord 75 IR and Perkin-Elmer Model 577 spectrometers.

Molecular weights of polymers were determined by vapour pressure osmometer of Hewlet-Packard (Model 302 B).

Intrinsic viscosities of dichloromethane solutions of polymers were measured at $19^{\circ} \mathrm{C}$ using Ubbelohde viscometer.

NMR spectra of new monomers and intermediate products were detected on $20 \%$ $(\mathrm{w} / \mathrm{v})$ solutions in deuterochloroform or deuterated acetone with $80 \mathrm{MHz}$ apparatus of Tesla BS $487 \mathrm{C}$ and at $100 \mathrm{MHz}$ with JEOL Model JNM-NH-100 spectrometer. Chemical shifts were determined by referring to HMDS (hexamethylsiloxane) internal standard.

DSC measurements were carried out on Perkin-Elmer DSC-2 apparatus under an argon atmosphere with a heating and cooling rate of $10 \mathrm{~K} \mathrm{~min}^{-1}$, as a rule. The results of DSC measurements were correlated with the visual observations of the texture of the samples by means of hot-stage microscope equipped with a pair of cross polarizers.

The same microscope of Boëtius type (PHMK Dresden) was used for determination of melting points of the monomers used for syntheses.

\section{RESULTS AND DISCUSSION}

\section{General Properties of Polymers}

The composition and properties of Series I of copolyesters derived from the mixtures of DHBP and its modification containing - $\mathrm{C}-$

$$
\stackrel{\mathrm{CC}}{\mathrm{I}} \mathrm{H}_{2}
$$

linkage between the aromatic rings of mesogen (BDCE) are summarized in Table I. Results for Series II and III of polymers prepared from methyl-substituted bisphenols are listed in Table II.

As it is seen from both intrinsic viscosity and vapour pressure osmometry measurements, the molecular weights of all prepared polymers are fairly low. The smaller values for Series III in comparison with those for Series II seem to point out that the reactivity of DMeBP-2 with dichlorides is lower than DMeBP-3, since in all cases the conditions of the synthesis were the same.

The data also show, that the molecular weights of samples having various molecular structures are slightly different. Comparison of 
Preparation and Properties of Thermotropic Mesogenic Polyesters

Table I. Characteristics of polymers of Series I

\begin{tabular}{|c|c|c|c|c|c|c|c|c|c|c|}
\hline \multirow{3}{*}{ Polymer } & \multirow{2}{*}{\multicolumn{2}{|c|}{$\begin{array}{l}\text { Bisphenol composition } \\
\text { BDCE/DHBP } \\
\frac{\mathrm{mol} \%}{\mathrm{~s}}\end{array}$}} & \multirow{3}{*}{$\frac{\begin{array}{c}\text { Polymer } \\
\text { yield }\end{array}}{\%}$} & \multirow{3}{*}{$M_{n}^{\mathrm{b}}$} & \multicolumn{6}{|c|}{ Transition temperatures $/{ }^{\circ} \mathrm{C}$} \\
\hline & & & & & \multicolumn{2}{|c|}{ Microscope } & \multicolumn{4}{|c|}{$\mathrm{DSC}^{\mathrm{c}}$} \\
\hline & $\begin{array}{l}\text { Of reaction } \\
\text { mixture }\end{array}$ & In polymer ${ }^{\mathrm{a}}$ & & & $T_{1}^{\prime}$ & $T_{2}^{\prime}$ & $T_{1}$ & $T_{2}$ & $T_{3}$ & $\Delta T=T_{3}-T_{2}$ \\
\hline $\mathrm{BDCE}+\mathrm{SEB}$ & $100 / 0$ & $100 / 0$ & 83.9 & 3200 & - & - & $\begin{array}{c}45 \\
(43)^{\mathrm{d}}\end{array}$ & - & - & - \\
\hline $\begin{array}{l}\text { BDCE } 70+ \\
\text { SEB }\end{array}$ & $70 / 30$ & $75 / 25$ & 79.2 & 2400 & 57 & 115 & $\begin{array}{c}30 \\
(22)\end{array}$ & $\begin{array}{c}69 \\
(57)\end{array}$ & $\begin{array}{l}120 \\
(94)\end{array}$ & $\begin{array}{c}51 \\
(37)\end{array}$ \\
\hline $\begin{array}{l}\text { BDCE } 50+ \\
\text { SEB }\end{array}$ & $50 / 50$ & $60 / 40$ & 81.3 & 2100 & 65 & 125 & $\begin{array}{c}27 \\
(21)\end{array}$ & $\begin{array}{c}80 \\
(57)\end{array}$ & $\begin{array}{c}122 \\
(102)\end{array}$ & $\begin{array}{c}42 \\
(45)\end{array}$ \\
\hline $\begin{array}{l}\text { BDCE } 30+ \\
\text { SEB }\end{array}$ & $30 / 70$ & $40 / 60$ & 83.9 & 1500 & 59 & 130 & $\begin{array}{c}48 \\
(10)\end{array}$ & $\begin{array}{c}78 \\
(70)\end{array}$ & $\begin{array}{c}121 \\
(112)\end{array}$ & $\begin{array}{c}43 \\
(42)\end{array}$ \\
\hline $\mathrm{DHBP}+\mathrm{SEB}$ & $0 / 100$ & - & 74.0 & $-^{\mathrm{e}}$ & 105 & 140 & - & $\begin{array}{c}126 \\
(121)\end{array}$ & $\begin{array}{c}143.5 \\
(-)\end{array}$ & $\begin{array}{l}17.5 \\
(-)\end{array}$ \\
\hline $\mathrm{DHBP}+\mathrm{SUB}$ & $0 / 100$ & - & 71.0 & $-^{\mathrm{f}}$ & 124 & 172 & - & $\begin{array}{c}126 \\
(125)\end{array}$ & $\begin{array}{l}164 \\
(-)\end{array}$ & $\stackrel{38}{(-)}$ \\
\hline $\mathrm{BDCE}+\mathrm{SUB}$ & $100 / 0$ & $100 / 0$ & 69.6 & 2900 & 59 & - & $\begin{array}{c}63 \\
(55)\end{array}$ & - & - & - \\
\hline
\end{tabular}

a Determined from $\mathrm{Cl}$ content in polymer sample.

b Measured by vapor pressure osmometry.

c Taken as the maximum of the transition peak.

d On cooling.

e Not soluble in $\mathrm{CHCl}_{3}, \eta_{\text {inh }}=0.191\left(\mathrm{dl} \mathrm{g}^{-1}\right)$ measured on $0.5 \%(\mathrm{w} / \mathrm{v})$ solution in $o$-chlorophenol at $25^{\circ} \mathrm{C}$.

${ }^{\mathrm{f}} \eta_{\mathrm{inh}}=0.193\left(\mathrm{dl} \mathrm{g}^{-1}\right)$, the conditions the same as above.

DSC results for two polymers differing in molecular weights (namely BDCE $50+$ SEB of $M_{n}=2100$ and 3700 and DMeBP-2/1 of $M_{n}=1790$ and 3080) indicated negligible effect of molecular weight on the transition temperatures (at least within this ranges of molecular weights). For BDCE 50+SEB samples we have obtained the following values for the temperatures of transition from crystalline to nematic phase and mesophase to isotropic liquid: $80,122^{\circ} \mathrm{C}$ and $80,122^{\circ} \mathrm{C}$ (respectively for $M_{n}=2100$ and $M_{n}=3700$ ). The other polymer (DMeBP-2/1) shows only one transition and its temperature is $54^{\circ} \mathrm{C}$ for the sample of $M_{n}=1790$ and $52^{\circ} \mathrm{C}$ for $M_{n}=3080$. It can be concluded, therefore, that the differences in liquid crystalline behaviour of polymers which are reported further are due to changes in molecular structure rather than to any variation of the molecular weights of the samples.
Liquid Crystalline Behaviour of Series I. of Copolyesters

DSC measurements and visual observations on a hot-stage microscope showed that all polyesters of Series I, except those in which the mesogen was built up from bisphenol containing $-\mathrm{C}-$ linkage between the aromatic rings (see data for BDCE + SEB or SUB in Table I), do behave as thermotropic nematics.

On DSC curves three transition temperatures (denoted in Table I as $T_{1}, T_{2}, T_{3}$ ) can be distinguished. Typically, those peaks were detected on heating and cooling as well, though in this last case some supercooling (usually $10-20 \mathrm{~K}$ ) was observed, especially for transitions at $T_{3}$ and $T_{2}$ (in Table I transitions on cooling are given in parantheses).

By comparing the DSC scans with the microscopic observations we have assigned $T_{2}$ 
Table II. Characterization of copolymers of Series II (from DMeBP-3) and III (from DMeBP-2)

\begin{tabular}{|c|c|c|c|c|c|c|c|c|c|c|c|c|}
\hline \multirow{3}{*}{ Series } & \multirow{3}{*}{ Polymer } & \multirow{2}{*}{\multicolumn{2}{|c|}{$\frac{\begin{array}{c}\text { Dichlorides } \\
\text { composition }^{\mathrm{a}}\end{array}}{\mathrm{mol}_{\mathrm{o}}^{\mathrm{o}}}$}} & \multirow{3}{*}{$\begin{array}{c}\begin{array}{c}\text { Polymer } \\
\text { yield }\end{array} \\
\%\end{array}$} & \multirow{3}{*}{$\frac{[\eta]^{\mathrm{b}}}{\mathrm{dl} \mathrm{g}^{-1}}$} & \multirow{3}{*}{$\mathbf{M}_{n}{ }^{\mathrm{c}}$} & \multicolumn{6}{|c|}{ Transition temperatures $/{ }^{\circ} \mathrm{C}$} \\
\hline & & & & & & & \multicolumn{2}{|c|}{ Microscope } & \multicolumn{4}{|c|}{$\mathrm{DSC}^{\mathrm{d}}$} \\
\hline & & SEB & SUB & & & & $T_{1}^{\prime}$ & $T_{2}^{\prime}$ & $T_{1}$ & $T_{2}$ & $T_{\mathrm{k}}^{\mathrm{e}}$ & $\Delta T^{\mathrm{f}}$ \\
\hline \multirow{6}{*}{ II } & DMeBP-3/1 & 100 & 0 & 80.1 & 0.28 & 4530 & 55 & 99 & 58 & 100 & 27 & 42 \\
\hline & DMeBP-3/2 & 80 & 20 & 79.9 & 0.25 & 3890 & 52 & 77 & 53 & 77 & 24 & 24 \\
\hline & DMeBP-3/3 & 60 & 40 & 80.9 & 0.45 & 6600 & 33 & 70 & 34 & g & 31 & (73) \\
\hline & DMeBP-3/4 & 50 & 50 & 79.5 & 0.33 & 5100 & 40 & 80 & 36 & 141 & 34 & 105 \\
\hline & DMeBP-3/5 & 30 & 70 & 76.7 & 0.16 & 3230 & 39 & 64 & 34 & 61 & 31 & 33 \\
\hline & DMeBP-3/6 & 0 & 100 & 68.2 & 0.26 & 4500 & 72 & 100 & 54 & 95 & 42 & 41 \\
\hline \multirow{3}{*}{ III } & DMeBP-2/1 & 0 & 100 & 78.8 & 0.21 & 3080 & - & 54 & - & 52 & - & - \\
\hline & DMeBP-2/2 & 50 & 50 & 72.2 & 0.11 & 2360 & 20 & 43 & 18 & $\mathrm{~h}$ & - & (69) \\
\hline & DMeBP-2/3 & 100 & 0 & 76.6 & 0.114 & 2440 & 48 & 70 & 50 & 71 & - & 21 \\
\hline
\end{tabular}

a In reaction mixture.

b Measured on $\mathrm{CH}_{2} \mathrm{Cl}_{2}$ solutions at $19^{\circ} \mathrm{C}$.

c Determined by vapor pressure osmometry on $\mathrm{CHCl}_{3}$ solutions.

d Taken as the maximum of the transition peak.

e On cooling.

f Temperature range of mesophase as determined by DSC.

g Broad endotherm, centered at $107^{\circ} \mathrm{C}$.

${ }^{\text {h }}$ Broad endotherm, centered at $87^{\circ} \mathrm{C}$.

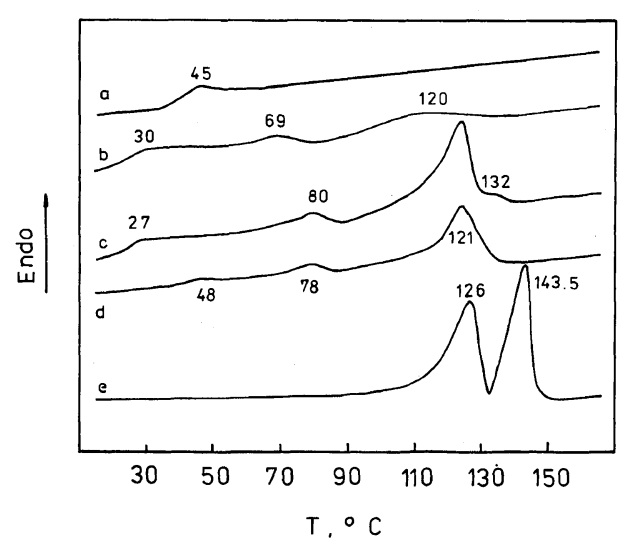

Figure 1. DSC curves for polymers of Series I synthesized from SEB and the following bisphenol compositions in the reaction mixtures: $\mathrm{a}, \mathrm{BDCE} / \mathrm{DHBP}=$ $100 / 0 ; \quad b, \quad B D C E / D H B P=70 / 30 ; \quad c, \quad B D C E / D H B P=$ $50 / 50 ; \mathrm{d}, \mathrm{BDCE} / \mathrm{DHBP}=30 / 70 ; \mathrm{e}, \mathrm{BDCE} / \mathrm{DHBP}=$ $0 / 100$.

and $T_{3}$ to crystalline-liquid crystalline $\left(T_{\mathrm{m}}\right)$ and mesophase-isotropic liquid $\left(T_{\mathrm{i}}\right)$ transitions. The lowest temperature transition $\left(T_{1}\right.$ in
Table I) which was not observed in the microscope, comes from $T_{\mathrm{g}}$ of the system and even most probably from its part enriched in BDCE + SEB blocks. The comparison of the elemental analysis data of the copolyesters of Series I and the composition of bisphenols taken to the synthesis showed that all polymers prepared from mixed bisphenols (DHBP+ $\mathrm{BDCE})$ are enriched in the one containing chlorine atom (that is BDCE). As a matter of fact, we have noticed in these cases that the part of DHBP was not used in the reaction course. It is, therefore, very likely that BDCE reacts faster and partly produces homopolyester blocks, which act as separate microphase (the quantitative agreement between the amount of DHBP left unreacted and the concentration of BDCE in copolyester was very good).

DSC and microscopically detected transition points are collected in tables to stress that both methods are complementary and 


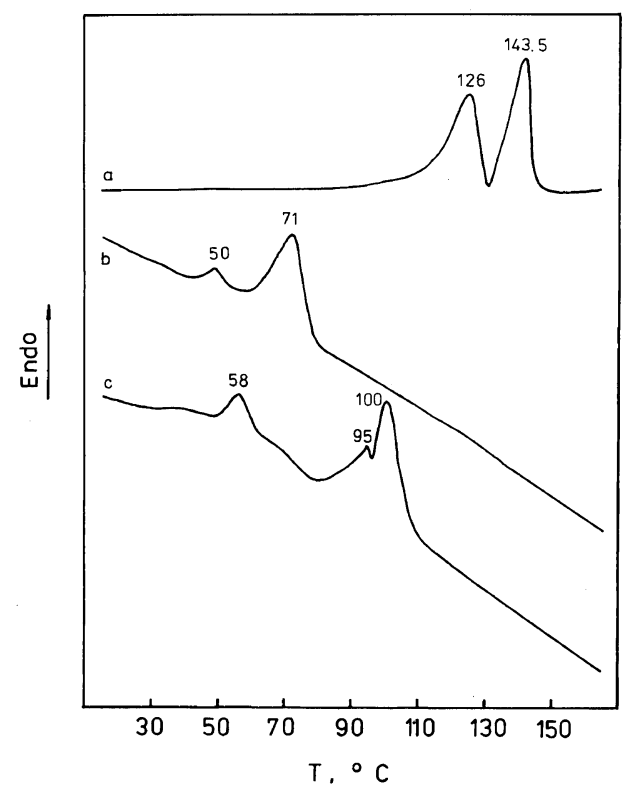

Figure 2. DSC scans for polyesters obtained from SEB with DHBP (a) and DHBP modified with lateral- $\mathrm{CH}_{3}$ group in position 2 (DMeBP-2, b) and 3 (DMeBP-3, c) of aromatic ring.

useful in the studies of liquid crystalline polymers. As it is seen, the agreement between the data (in Table I $T_{1}^{\prime}$ corresponds to $T_{2}$ and $T_{2}^{\prime}$ corresponds to $T_{3}$ ) is quite good, though in the case of Series I the glass transition could not be microscopically observed.

As it was mentioned above, BDCE in polycondensation with SEB or SUB does not give polyesters of liquid crystalline properties. When it was mixed, however, with the bisphenol which itself yields the liquid crystalline material (as DHBP), BDCE did not reduce the ability of copolyester to exhibit the mesophasic behaviour, but on the contrary broadened the range of liquid crystalline phase $(\Delta T)$. For $\mathrm{DHBP}+\mathrm{SEB}$ polyester $\Delta T$ was only $17.5^{\circ} \mathrm{C}$, while for the copolyesters of mixed bisphenols (DHBP + BDCE) about $40^{\circ} \mathrm{C}$, depending on the molar ratio of DHBP to BDCE. For these latter polymers, however, the appearance of mesophase was much shifted towards lower temperatures. From the data of Table I, it can be seen, that the temperatures of the transi-

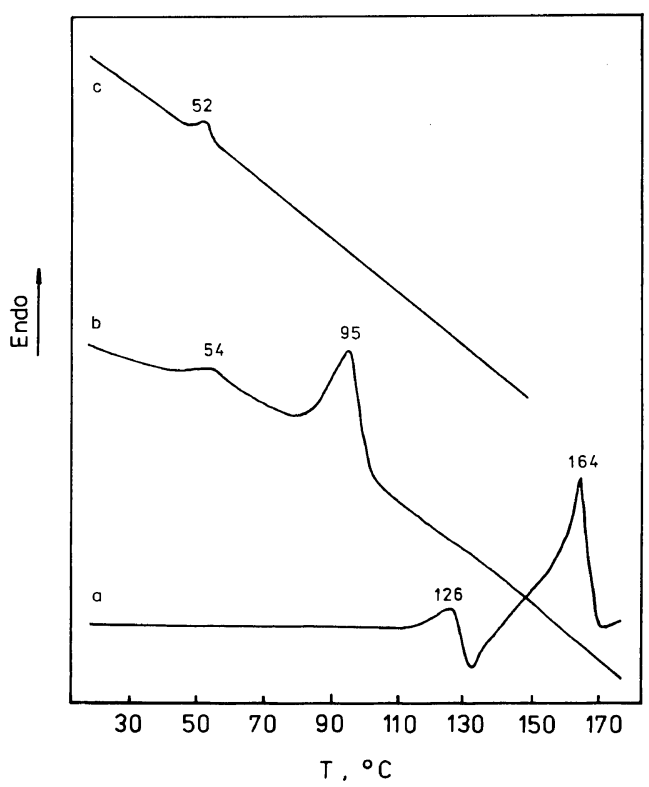

Figure 3. DSC curves for polyesters derived from SUB with DHBP (a) and its modified analogues having $-\mathrm{CH}_{3}$ group in position 3 (b) and 2 (c) towards ketone linkages.

tion from crystal to mesophase $\left(T_{2}\right)$ for the studied copolyesters of mixed bisphenols $(\mathrm{DHBP}+\mathrm{BDCE})$ were reduced more with respect to the value for polyester of $\mathrm{DHBP}+\mathrm{SEB}$, than the temperatures of the transition from mesophase to isotropic liquid $\left(T_{3}\right)$. Nevertherless, in both cases, we did not observe the strong relationship between the composition of bisphenols and the location of $T_{2}$ and $T_{3}$ along the temperature axis. The reduction of DSC peaks heights with the increase of BDCE content in the copolymer was, however, quite remarkable.

\section{Liquid Crystalline Properties of Series II and III Copolyesters}

From DSC curves for Series II and III two transition peaks (denoted as $T_{1}$ and $T_{2}$ in Table II) could be determined for the heating cycle but only one (corresponding to $T_{1}$ ) for cooling. The visual observations with the polarized microscope allowed us to assign the lower temperature peak $\left(T_{1}\right)$ to the melting, 


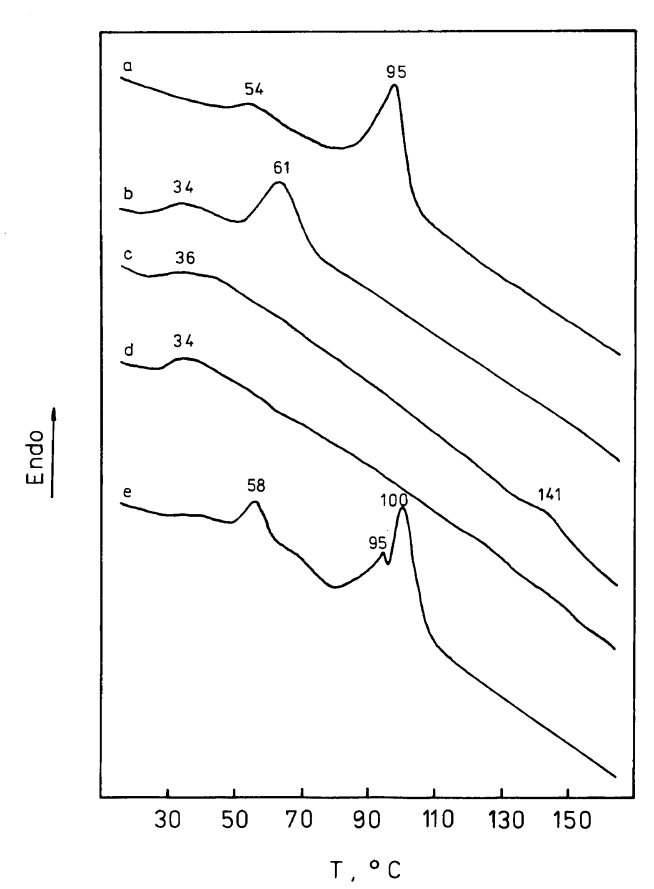

Figure 4. DSC curves for Series II of polymers prepared from DMeBP-3 and different ratios of SUB/SEB: a, 100/0; b, 70/30; c, 50/50; d, 40/60; e, 0/100.

that is the transition from the crystalline to nematic phase (usually abbreviated as $T_{\mathrm{m}}$ ) and $T_{2}$ to the isotropisation transition $\left(T_{\mathrm{i}}\right)$. The absence of $T_{\mathrm{i}}$ transition on DSC scans for the cooling cycle is not an extraordinary phenomenon and was observed by many scientists, ${ }^{16,17}$ also for polyesters. ${ }^{18,19} \mathrm{We}$ have faced it in our earlier studies as well.

Actually the transition peaks of copolymers as determined by DSC are usually much less defined than those of the homopolymers ${ }^{20}$ (in our case this corresponds to polyester of one type of bisphenol or dicarboxylic acid dichloride). For copolyesters, the broad and small melting endotherms are fairly common, especially for the compositions containing equimolar or close to it ( 40 or $60 \mathrm{~mol} \%$ ) amount of bisphenol or dichloride in the repeating unit. It is clearly seen for the copolyesters of Series II or III, obtained from 60/40 (sample of DMeBP-3/3) or 50/50 (sample of DMeBP-2/2) $\mathrm{mol} \%$ of SEB/SUB in the reaction mixture.
On DSC scan for DMeBP-3/3 polymer two peaks are present, but since the upper temperature endotherm is very broad and shallow (from 82 to $147^{\circ} \mathrm{C}$ ) the exact transition temperature could not be determined. The same situation occurred for DMeBP-2/2 copolyester (temperature range from 47 to $107^{\circ} \mathrm{C}$ ). We must add that all data reported in tables were obtained on unannealed samples of the asprepared polymers during the first heating cycle.

Considering the above mentioned difficulties in exact determination of isotropisation temperatures for those two samples, in Table II we did not insert their values, and the temperature range of liquid crystalline phase is given in parentheses, taking as reference isotropisation temperature the center of broad endotherm.

The microscopic observations did not help much either. Apparently due to the low degree of crystallinity of those two copolymers, the pattern were rather dark, so it was almost impossible to detect precisely the transition from mesophase to isotropic liquid.

In view of the microscopic and DSC results we think, the behaviour of Series II and III copolymers containing mixed flexible spacers, could be explained assuming the existence of partly random or block copolyesters in the system. Such copolymers would be expected to exhibit a biphasic region in which both mesophasic and isotropic states coexist. It is very likely then, that segments built up from bisphenol and longer flexible unit (that is sebacoyl dichloride) would undergo isotropisation transition in lower temperature thus being already in isotropic state, while those of shorter aliphatic chain (from suberoyl dichloride) are still liquid crystalline. One can imagine also, that for some ratios of SEB/SUB in the system, the liquid crystalline arrangement will not be possible at all. We believe that the same effect may occur in the case of distribution of the lengths of mesogenic fragments. The similar observations, but for polyesters prepared by esterification were earlier reported by 
Krigbaum et al. ${ }^{20}$

As it is seen from Table II, the condensation of SEB and/or SUB with bisphenol modified with lateral $\mathrm{CH}_{3}$-substituent (DMeBP-2 or DMeBP-3) generally leads to polyesters of lower transition temperatures (both $T_{\mathrm{m}}$ and $T_{\mathrm{i}}$ ) and broadened temperature range of liquid crystalline phase. However, depending on the position of substitution the various shifts of the temperature of appearance of nematic phase were observed. From our results it can be concluded, that the substitution of the aromatic ring in position 2 , as referred to $-\mathrm{C}_{11}-$

linkage, is more effective in lowering $T_{\mathrm{m}}$ and $T_{\mathrm{i}}$ (polyesters of DMeBP-2) than in position 3 (polyesters of DMeBP-3). This is in agreement with the studies by Blumstein et al. ${ }^{5}$ on polymers with mesogens modified with two lateral $\mathrm{CH}_{3}$ groups.

Among polymers of Series II and III, there are two which behave exceptionally. Copolyester derived from methyl-substituted bisphenol with equimolar ratio of SEB and SUB (sample DMeBP-3/4) exhibited the extremely high value of isotropisation temperature $\left(T_{2}=141^{\circ} \mathrm{C}\right)$ and on DSC curve instead of broad peak, the sharp, though of low energy, transition appeared. For the moment it is rather difficult to explain such increase in $T_{\mathrm{i}}$ and, consequently the broadening of the temperature range of liquid crystalline behaviour. It is, however, possible that for this particular composition, the space filling of the macrochains is favoured leading to a more ordered packing. As a result the increase in isotropisation transition is to be expected. The similar effect, that is broadening of $\Delta T$ for equimolar copolyesters having mixed polymethylene spacers, was observed by Ober et $a^{21}$

On the other hand, on DSC scans for polyester obtained from DMeBP-2 with SUB one sharp peak at $52^{\circ} \mathrm{C}$ was detected. Under polarized microscope in this temperature darkening of already dim (due to the low degree of crystallinity) microscopic image was observed. It is, therefore, reasonable to presume, that this temperature marked the beginning of the transition to isotropic melt. Two facts, the dark image of the sample in the polarized microscope and the absence of peaks on DSC curves on cooling are in favour to assume that the polyester of $\mathrm{DMeBP}-2+\mathrm{SUB}$ does not exhibit the liquid crystalline properties. The disappearance of mesophasic behaviour with the modification of mesogen by lateral substitution is not unique and has been previously observed by others, though for polar chlorine substituent. ${ }^{6}$

As for the mesophasic ranges $(\Delta T)$ for polyesters having mesogens laterally modified is concerned, they also depend on the position of substitution. In series with suberoyl dichloride as flexible segment, the methyl substitution of aromatic ring in position 3 with respect to ketone linkage (DMeBP-3+SUB) practically does not change $\Delta T$ in comparison with the value for polyester derived from unsubstituted bisphenol $\left(41\right.$ and $38^{\circ} \mathrm{C}$, respectively). On the contrary, as it was mentioned earlier, 2-substituted bisphenol does not yield at all the mesomorphic polyester (compare data for DMeBP-2 + SUB in Table II).

In the series of sebacoyl dichloride polyesters, the increase of $\Delta T$ has been observed for both 3- and 2-substituted derivatives, though much more pronounced for polyesters obtained from $3\left(42^{\circ} \mathrm{C}\right)$ than 2-substituted $\left(21^{\circ} \mathrm{C}\right)$ bisphenols. For comparison, $\Delta T$ for $\mathrm{DHBP}^{+}+$ SEB was only $17.5^{\circ} \mathrm{C}$. Here again the controlling effect of the length of the flexible unit on the thermal properties of polyesters was additionally demonstrated.

The results of our work support the conclusion, that in thermotropic polyesters having stiff (mesogenic) and flexible segments in the main chain, lateral substitution as well as replacement of the ketone linkage by $-\mathrm{C}-$<smiles>[GeH3]</smiles>
group produce very strong effects on mesogenic properties, consisting in shift of the 
transition temperatures and alteration of temperature range of liquid crystalline phase. Similar effects were also observed with the change of the lengths of the flexible segments.

\section{REFERENCES}

1. A. Blumstein, J. Asrar, and R. B. Blumstein, Liq. Cryst. Ordered Fluids, 4, 311 (1984).

2. R. W. Lenz and J. I. Jin, Liq. Cryst. Ordered Fluids, 4, 347 (1984).

3. L. Makaruk and H. Polańska, Liq. Cryst. Ordered Fluids, 4, 154 (1984).

4. L. Makaruk, H. Polańska, and B. Ważyńska, "Polymeric Liquid Crystals," A. Blumstein Ed., Plenum Publishing Corporation, N. Y., 1985, pp 265-274.

5. A. Blumstein, S. Vilasagar, S. Ponratham, S. B. Clough, R. B. Blumstein, and G. Maret, J. Polym. Sci., Polym. Phys. Ed., 20, 877 (1982).

6. S. Antoun, R. W. Lenz, and J. I. Jin, J. Polym. Sci., Polym. Chem. Ed., 19, 1901 (1981).

7. G. Galli, E. Chiellini, C. Ober, and R. W. Lenz, Makromol. Chem., 183, 2693 (1982).

8. Qi-Feng Zhou and R. W. Lenz, J. Polym. Sci., Polym. Chem. Ed., 21, 3313 (1983).

9. W. J: Jackson, Br. Polym. J., 12, 154 (1980).
10. C. Noël, C. Friedrich, F. Laupretre, J. Billard, L. Bosio, and C. Strazielle, Polymer, 25, 363 (1984).

11. S. Porejko and Z. Wielgosz, Polimery, 13, 55 (1968).

12. B. Bochwic, "A Textbook of Practical Organic Chemistry," Polish Scientific Editing Company (PWN), Warsaw, 1975, p 208.

13. A. T. Carpenter and R. F. Hunter, J. Appl. Chem., 3, 490 (1953).

14. L. Makaruk, H. Polańska, and E. Księżakowska, in preparation.

15. J. A. Riddick and E. F. Toops, "Organic SolventsPhysical Properties and Methods of Purification in "Techniques of Organic Chemistry," Vol. VII, A. Weissberger, Ed., Interscience Publishers, Inc., New York, N. Y., 1955.

16. K. Iimura, N. Koide, H. Tarabe, and M. Takeda, Makromol. Chem., 182, 2569 (1981).

17. G. Galli, P. Nieri, C. Ober, and E. Chiellini, Makromol. Chem., Rapid Commun., 3, 548 (1982).

18. K. Iimura, N. Koide, R. Ohta, and M. Takeda, Makromol. Chem., 182, 2563 (1981).

19. G. Galli, H. Laus, A. S. Angeloni, P. Ferruti, and E. Chiellini, Makromol. Chem., Rapid Commun., 4, 681 (1983).

20. W. R. Krigbaum, R. Kotek, T. Ishikawa, and H. Hakemi, Eur. Polym. J., 20, 225 (1984).

21. C. Ober, J. I. Jin, and R. W. Lenz, Polym. J., 14, 9 (1982). 